\title{
Promotion of cooperation when benefits come in the future: A water transfer case
}

\author{
December 12, 2016
}

\begin{abstract}
This paper presents a two-regime differential game, with a first period in which two countries cooperate in a joint investment project to construct a specific infrastructure. This period ends when the infrastructure is finished, which serves to increase each player's welfare in a subsequent non-cooperative game played by the two countries thereafter. We define an imputation distribution procedure (IDP) to share the investment costs during cooperation according to each player' future benefits. We prove that the IDP is time consistent if at any time within the cooperative period each country's share on the surplus to go is equal to or converges towards the country's relative gains from the existence of the infrastructure (realized in the subsequent non-cooperative period). Furthermore, we obtain the instantaneous side-payment scheme which makes the IDP feasible. The mechanism is studied for a joint investment project to build a water canal to transfer water between a surplus and a deficit river basin.

JEL Classification: F18, C73.
\end{abstract}

Keywords: Cooperative differential game, non-cooperative differential game, imputation distribution procedure, instantaneous side-payment, time-consistent solution.

\section{Introduction}

This paper proposes a time consistent distribution procedure to insure cooperation in a joint investment project between to countries or regions when the benefits will come when the infrastructure is finished. To situate the problem we refer to a a particular application, the construction of a canal for water transfer. In this context our paper analyzes the dynamic cooperation between two countries or regions in order to build a canal which connects a donor river basin, with higher precipitation rates, and a recipient river basin, with greater water productivity. This joint investment program presents two main characteristics. First, cooperation does not lead to an immediate reward, but only after an initial period in which the two parts have to pay the costs of building the canal. Secondly, the delayed benefits of the cooperation are known by the cooperating agents and they are typically asymmetric. The efficiency gains linked to the flow of water from a surplus basin to a deficit basin with greater water productivity, can be realized thanks to the water market created by the water canal. 
Some examples of already operative schemes or ongoing projects of water transfer can be found, usually within a specific country: ${ }^{1}$ The Tagus-Segura Transfer Project in Spain, the Snowy River Scheme in Australia, the São Francisco Interlinking Project in Brazil, the Olmos Transfer Project in Peru or the South-North Water Transfer Project in China. Some of these are ongoing projects, whose full implementation may take some decades, or even end-up as fail projects. These projects have been promoted by a central government. Much less frequent are the examples of water transfers between different countries, like the transfer from the Kosi river in Nepal to the Ganges in India and Bangladesh, or the Lesotho Highlands Water Project (drawn out by corruption) between Lesotho and South Africa (geographically condemned to get along with each other). This reflects the difficulties tied to the obliged cooperation between two governments who have to determine how to share the costs of the joint project, and how to distribute these costs along the often lengthy construction period. Maybe better examples of these difficulties are the failed projects, like the Rhone-Barcelona aqueduct proposed to supply the city of Barcelona in Spain with water from the Rhone river in France (see, Lopes (2008)). We will focus on the economic aspects that help maintain the agreement to build the canal although, as pointed out by Lopes (2008), the obstacles to the transfer between countries are also political and institutional.

The bulk of the literature on river water management involving two regions/countries and non-cooperative game theory is on water-sharing under an upstream/downstream configuration (see, for example, Ambec and Ehlers (2008), Bhaduri and Barbier (2008), Ambec and Sprumont (2002), or Kilgour and Dinar (1995, 2001)). However, the problem of a water transfer between two river basins has some specificities not present in the upstream/downstream literature. The donor river basin must be characterized by a surplus of water inflows, and the recipient basin by a deficit. Given this asymmetry, water productivity is higher in the recipient. Two additional features are also present in most of the examples highlighted above. The recipient may have access to alternative sources of water, by investing in infrastructures which can help to increase the available water for the economy (examples could be desalination plants, projects to save water, to reduce pipeline leaking, or investment in recycling). The transfer brings environmental consequences mainly, but not exclusively, for the donor. If the relative cost of the alternative water supplies is low, and/or the magnitude of the environmental damages linked to the water transfer is high, then the inter-basin transfer would not be profitable.

For the particular example of the Tagus-Segura transfer, Ballestero (2004) presents a static demand-supply model, later extended to a dynamic setting in Cabo et al. (2014). The latter studies the interaction between a donor and a recipient region as a non-cooperative differential game, which defines the water market as a bilateral monopoly. It also includes the environmental damage in the donor region caused by the transfer, and the alternative water supplies available for the recipient. It is assumed nonetheless, that the infrastructure required to transfer the water between the two river basins is already operative. ${ }^{2}$ Under

\footnotetext{
${ }^{1}$ In some of these projects water is transferred from one river basin to another river, others transfer the water to dams in the mountains (for irrigation and to generate hydroelectricity), or towards a specific region for municipal water supply, industry and irrigation.

${ }^{2}$ For this case study, $230 \mathrm{~km}$ network of canals, aqueducts and tunnels were built by the Spanish central government to transfer water from the Tagus basin in the center of Spain to the Segura basin in south-eastern Spain.
} 
this assumption the water market equilibrium is dynamically analyzed. By contrast, with a broader perspective, and particularly when the transfer involves governments from two different countries, we consider it important to address the previous coordination problem associated with the joint investment required to build the canal.

The central question in static cooperative game theory of how to distribute the gains from cooperation between the cooperating players, is extended by dynamic cooperative game theory to study the distribution of these gains not only among players but also over time. ${ }^{3}$ In particular, how to distribute the surplus from cooperation over time to guarantee that no player has an incentive to deviate from cooperation, at any point in time (the cooperative payoffs to go surpass the non-cooperative payoffs to go at any time within cooperation). This concept is usually referred to as time consistency. ${ }^{4}$ One widespread mechanism to guarantee the time consistency of the cooperative solution is to select a solution concept specifying each player's share of the total cooperative payoff, and define a payoff distribution procedure, as stated in Petrosjan (1997), to decompose the individual total payoff over time, in such a way that time consistency is preserved (see Zaccour (2008) for a review). This mechanism constitutes the basis for implementing a time-consistent solution in this paper, although the literature provides other mechanisms. ${ }^{5}$

In our setting cooperation does not lead to immediate gains in payoffs. On the contrary, cooperation to invest in the construction of the canal, represents a costs for both players, maintained throughout the period of cooperation. In fact, the gains from the cooperation only start once the water starts to flow through the canal, and with it come the efficiency gains. But this is precisely the exact moment at which the joint investment cooperation halts. Therefore, the first question that must be addressed is how to share the "costs" of cooperation when its benefits will only materialize when the cooperation ceases to exist, and the two parts engage in a non-cooperative trade relationship in the water market. Thus, assuming that the aggregated (discounted) gains from the existence of the water canal surpasses the global economic and environmental costs of the joint investment project, our main research question is: how should the investment costs be shared between the two parts and distributed over time to guarantee the time consistency of the cooperate solution? That is, to guarantee that no player deviates from the cooperation and the canal is actually finished. We would like to stress that our model shares some similarities with the holdup problem analyzed in the literature (see, for example the dynamic formulation of this problem in Che and Sákovics 2004). Both problems seek to maintain a joint investment project over time in order to enjoy a future surplus. Nonetheless the two approaches attack this problem from a different perspective. From a non-cooperative perspective, the holdup problem seeks a bargaining solution which divides the future surplus between the two players in order to guarantee current investment. By contrast, in a cooperative framework, we define a sharing mechanism to distribute current investment costs (taking into account future benefits) which

\footnotetext{
${ }^{3}$ For an upstream/downstream pollution problem, Jørgenssen and Zaccour (2001) propose an instantaneous side-payment scheme to share the current surplus from an agreement to reduce downstream pollution.

${ }^{4}$ As stated in Zaccour (2008), it has been also called sustainability of cooperation, dynamic individual rationality, dynamic stability, durability of an agreement, or agreeable solution.

${ }^{5}$ Other mechanisms can be found in the literature, like the incentive strategies proposed by Ehtamo and Hämaläinen $(1986,1989,1993)$, or the design of the cooperative agreement to satisfying the property of being at equilibrium (see, for example Rincón-Zapatero et al. (2000)).
} 
satisfies the property of time consistency.

To analyze this question we define a differential game with two different regimes. The two countries jointly invest to build the canal within a first period, whose length will be determined by the intensity of the investment paths. ${ }^{6}$ The cooperative objective function includes current investment costs as well as future benefits; the latter in the form of a scrap function defined as the sum of the value functions of the two players in the subsequent game. Within this subsequent period of infinite length the canal is operating and the two countries play a non-cooperative differential game, as in Cabo et al. (2014). By comparing the latter with a baseline scenario of no transfer, we observe asymmetric surpluses for the two players from the existence of the canal. Therefore, we can compute each player's share in the total gains stemming from the existence of the canal. This share helps us to link current investment costs during cooperation and future benefits. The cooperative solution concept proposed in this paper is based on the central idea that at each time within cooperation, each player's payoffs to go must be equal to his payoffs to go in case of defection (and hence no transfer), plus a share of the total surplus to go. Equivalently, at each time within cooperation, the excess gains of a player if cooperation is maintained in relative terms (to the total surplus to go) must be positive and must converge towards this player's share in total gains from the existence of the canal (computed at the moment when the canal starts to be operative). In consequence, the proposed method assigns each cooperating player a contribution (to the joint investment required to build the canal) dependent on his share in total gains (obtained in the second period when the canal is operative). With this principle we are able to define an Imputation Distribution Procedure (IDP) specifying what each player should be contributing at each moment through the cooperative period in order to guarantee time consistency. Furthermore, this time-consistent IDP is feasible. At any time within cooperation, the instantaneous payoff assigned by the IDP to the two countries adds up to the instantaneous joint cooperative payoff. Therefore, we can compute the instantaneous transfer scheme throughout the cooperative period that makes this IDP possible.

The paper is organized as follows. Section 2 describes the problem and presents the model. Section 3 presents the main contribution of the paper: the definition of an imputation distribution procedure that guarantees the time consistency of the cooperative solution and which can be attained through an instantaneous side-payment scheme. Section 4 shows our results for a linear quadratic example. Section 5 concludes.

\section{The model}

This section describes a two-regime differential game between a (donor) country/region which river basin is characterized by relatively high precipitation rates and relatively low productivity of water and a (recipient) country/region with lower precipitations and highly productive uses. If the two countries cooperate in a first period and build a canal, this would make an inter-basin water transfer possible thereafter. In this water market the donor will transfer surplus water for a price, and it will experience environmental damage due to the

\footnotetext{
${ }^{6}$ Differential games have been used to analyze cooperation in $R \& D$, which increases the size of an $R \& D$ project and reduces the time to completion (see, for example, Kort and Navas (2007) and references therein.
} 
deterioration of its water quality. The recipient will pay the price for the water transfer which can be used to enhance production, and will also allow reductions in the investments on subsidiary water production, water savings or water recycling. We assume henceforth that the savings from lower investments on subsidiary water supplies plus the increments in production in the recipient surpass the environmental losses in the donor plus the total cost of building the canal, leading to a rise in the Kaldor-Hicks efficiency. This is a necessary condition for the regions to cooperate and build the canal.

We first present the main hypotheses to describe the economic and environmental aspects of the relationship connecting these two regions. Then we present the non-cooperative game played within a second period when the canal is already operative, and analyze the dynamic determination of quantity and price in the water market. Finally, we present the cooperative period when countries jointly invest to build the canal.

\subsection{The donor and the recipient}

This section follows the main assumptions in Cabo et al. (2014). Assuming no uncertainty, a constant surplus of water still remains in the donor region after covering demands. Without the canal this surplus flows through the donor's river basin. However, if an aqueduct is built, an amount $\tau(t)$ of the surplus could be transferred to the recipient. Before the transfer, the inhabitants in the donor region would enjoy the environmental amenities or environmental services of what we denote a pristine river. By contrast, the decrease in the water level provoked by the water transfer would decrease these amenities at an increasing rate. Thus, the environmental amenities can be represented by $E(\tau(t))$, with $E(0)>0, E^{\prime}(\tau(t))<0$ and $E^{\prime \prime}(\tau(t))<0$. As compensation the donor receives a monetary payment, $p(t)$, from the recipient for each unit of water transferred. The instantaneous welfare function for the donor is then expressed as ${ }^{7}$

$$
F^{d}(p(t), \tau(t))=E(\tau(t))+p(t) \tau(t) .
$$

Due to a relatively higher water productivity, the recipient will be willing to pay for the water transferred from the donor. Nevertheless, apart from the transfer, the recipient has an alternative and often subsidiary way to increase its volume of available water. It can invest in the equipment required either for water saving (reducing water use or water leakage from the distribution network), water recycling (use of gray water), or water production (like desalination plants). The usable water capacity, $x(t)$, is defined as the capacity to produce, recycle or save water using current equipment (measured in cubic meters). Capacity evolves according to:

$$
\dot{x}(t)=\sigma(t)-\delta x(t), \quad x(0)=x_{0} \geq 0,
$$

with $\delta>0$ being the depreciation rate and $\sigma(t)$ the investment to replenish and further increase current capacity.

Recipient's welfare comes from the amount of available water: either the water transferred, $\tau(t)$, or the usable water capacity, $x(t)$. Welfare increases with the amount of available water at a decreasing rate. Investments in new capacity are increasingly costly, which can reflect increasing transaction costs and/or incremental costs of successive projects to produce, save or recycle water. $Q(\tau, x)$ and $C(\sigma)$ denote respectively the gross benefit

\footnotetext{
${ }^{7}$ Here and henceforth, superscript $d / r$ refers to donor/recipient respectively.
} 
and the cost of investment. Finally, instantaneous welfare decreases with transfer payments to the donor. Hence, the welfare function of the recipient is expressed by:

$$
F^{r}(p(t), \tau(t), x(t), \sigma(t))=Q(\tau(t), x(t))-p(t) \tau(t)-C(\sigma(t))
$$

with $Q(\tau, x)$ concave in $\tau$ and $x, C^{\prime}(\sigma)>0$ and $C^{\prime \prime}(\sigma)>0$. For simplicity we assume that the water inflow in the recipient river basin is null. We believe that none of the results would be affected if instead, a constant inflow of water were assumed also in the recipient river basin.

The problem must be solved backwards. First, we present the non-cooperative differential game of infinite duration, starting at moment $T$, when the canal starts to be operative. The value functions of donor and recipient in the water market game, played within period $[T, \infty)$, must be taken into account to compute the cooperative solution of a joint investment program to build the canal within a first period $[0, T)$.

\subsection{The non-cooperative water market game within $[T, \infty)$}

This subsection describes the water market enabled by the existence of the canal. It presents the dynamic interaction between the donor and the recipient regions starting at time $T$ when the canal starts to be operative. The time paths for the amount and the price of the water transfer are determined from the supply and demand decisions taken by donor and recipient as described in Cabo et al. (2014). The donor determines the supply of water, $\tau^{d}$, in order to maximize the stream of welfare discounted at a constant rate, $\rho$, within an infinite time horizon: ${ }^{8}$

$$
\max _{\tau} \int_{T}^{\infty}[E(\tau)+p \tau] e^{-\rho(t-T)} d t
$$

Correspondingly, the recipient must decide on the demand for water, $\tau^{r}$, and on the investment, $\sigma$, in usable water capacity, to maximize discounted welfare:

$$
\begin{gathered}
\max _{\tau, \sigma} \int_{T}^{\infty}[Q(\tau, x)-p \tau-C(\sigma)] e^{-\rho(t-T)} d t, \\
\text { s.t.: } \dot{x}(t)=\sigma(t)-\delta x(t), \quad x(T)=x_{T} \geq 0 .
\end{gathered}
$$

The recipient is a farsighted player whose maximization problem is subject to the evolution of usable water capacity in (5). In contrast, the donor behaves as a static or myopic player. ${ }^{9}$ Price is determined by the optimal supply and demand decisions and by the Market clearing condition:

$$
\tau^{d}(p)=\tau^{r}(p)
$$

Assuming that a feedback Nash equilibrium for this problem exists, ${ }^{10}$ we denote with $V^{i}\left(x_{T}\right)$ the value function of player $i \in\{d, r\}$, with an initial stock of usable water capacity given by $x(T)=x_{T}$. To gain an insight of each player's incentive to invest in the construction of the canal, these values must be confronted with each player's accumulated gains in the absence of an aqueduct to transfer the water, and under the assumption of an identical initial stock of usable water capacity, $x_{T}$.

\footnotetext{
${ }^{8}$ Here and henceforth, the time argument is omitted when no confusion arises.

${ }^{9}$ Problem (3) could be written as $\max _{\tau}[E(\tau)+p \tau]$.

${ }^{10}$ The game is analytically solved in Cabo et al. (2014) under a linear quadratic specification for functions $E(\tau), Q(\tau, x)$, and $C(s)$.
} 
With no water transfer, the donor would not face any optimization problem and his profit would read: ${ }^{11}$

$$
V_{N T}^{d}=\frac{F^{d}(0,0)}{\rho} .
$$

The recipient would choose the investment in usable water capacity to solve the optimal control problem:

$$
V_{N T}^{r}\left(x_{T}\right)=\max _{\sigma} \int_{T}^{\infty} F(0,0, x, \sigma) e^{-\rho(t-T)} d t=\max _{s} \int_{T}^{\infty}[Q(0, x)-C(\sigma)] e^{-\rho(t-T)} d t
$$

subject to $(5)$.

Condition 1 We first assume $e^{12}$ that there exists a set $A \subseteq R^{+}$such that

$$
V^{i}\left(x_{T}\right)>V_{N T}^{i}\left(x_{T}\right), \quad \forall x_{T} \in A, \quad i \in\{d, r\}
$$

Condition 1 states that both players are better off if the canal exists. This gives them both an incentive to cooperate in a joint investment project to build the canal.

\subsection{The joint investment project to build the canal within $[0, T)$}

Within a first period $[0, T)$, the two regions cooperate in a joint investment project to build the canal. At any time $t \in[0, T)$, each country $i$ invests an amount $I^{i}(t)$ at a cost $C^{i}\left(I^{i}(t)\right)$. The joint investment contributes to increase the length of the aqueduct already built, measured by the stock variable $K(t)$. The canal is finished once it reaches its full length, when the accumulated stock $K(t)$ reaches the value $\bar{K}$. Thus, the duration of this cooperative period, $T$, is determined by the intensity of investments, and by the magnitude of the canal represented by constant $\bar{K}$. Within this period, the cooperating agents must also decide the investment in alternative water supply, $\sigma(t)$. Taking into account the expectations of a future water transfer of infinite duration, the incentive to invest in the stock of usable water capacity is reduced. The cooperative maximization problem can be written as:

$$
\begin{aligned}
& \max _{I^{d}, I^{r}, \sigma, T} \int_{0}^{T}\left[F^{d}(0,0)+F^{r}(0,0, x, \sigma)-C^{d}\left(I^{d}\right)-C^{r}\left(I^{r}\right)\right] e^{-\rho t} d t+S C(x(T)), \\
& \text { s.t.: } \dot{x}=\sigma-\delta x, \quad x(0)=x_{0} \geq 0, \\
& \text { s.t.: } \dot{K}=I^{d}+I^{r}, \quad K(0)=K_{0}, \quad K(T)=\bar{K},
\end{aligned}
$$

where the scrap value, $S C(x(T))$, is given by the present value of the addition of the value functions of donor and recipient in the non-cooperative differential game played a la Nash from $T$ on. Considering feedback strategies, as presented in Subsection 2.2, the scrap value reads

$$
S C(x(T))=\left[V^{d}(x(T))+V^{r}(x(T))\right] e^{-\rho T} .
$$

Assuming that this problem has a solution, ${ }^{13}$ the value function of the cooperative game is denoted as

$$
V_{\mathrm{C}}\left(x_{0}\right)=\int_{0}^{T}\left[F^{d}(0,0)+F^{r}\left(0,0, x_{\mathrm{C}}, \sigma_{\mathrm{C}}\right)-C^{d}\left(I_{\mathrm{C}}^{d}\right)-C^{r}\left(I_{\mathrm{C}}^{r}\right)\right] e^{-\rho t} d t+S C\left(x_{\mathrm{C}}(T)\right),
$$

\footnotetext{
${ }^{11}$ Subscript NT refers to "no-transfer" scenario.

${ }^{12}$ For the linear quadratic game proposed in Cabo et al. (2014), Condition 1 is satisfied for $A=[1 / \alpha,+\infty)$.

${ }^{13}$ For the lineal quadratic case, the solution is computed in the Appendix.
} 
where $s_{\mathrm{C}}(t), I_{\mathrm{C}}^{d}(t)$ and $I_{\mathrm{C}}^{r}(t)$ are the optimal investment paths of the cooperative game (7)(9), and $x_{\mathrm{C}}(t)$ is the cooperative stock of usable water capacity which solves equation (8) for the optimal investment path $\sigma_{\mathrm{C}}(t)$. The level $x_{\mathrm{C}}(T)$, reached by this stock when the players cooperate from 0 till the moment $T$ at which the canal is finished, also defines the starting stock of usable water capacity for the subsequent non-cooperative water market game. Note that this value function collects the accumulated payoff of the two countries within the first cooperative period, plus what they get within the subsequent non-cooperative game, included in the scrap value $S C(x(T))$.

A necessary condition for the two players to agree to cooperate in the joint investment program (7)-(9) is:

Condition 2 Overall global rationality or Kaldor-Hicks efficiency:

$$
V_{C}\left(x_{0}\right)>V_{N T}^{d}\left(x_{0}\right)+V_{N T}^{r}\left(x_{0}\right) .
$$

The accumulated payoffs globally considered for the two players when they coordinate their efforts to build a canal within a first period and utilize it henceforth, $V_{\mathrm{C}}\left(x_{0}\right)$, must be greater than the addition of the accumulated payoffs for the two regions if the canal is never initiated and no transfer ever takes place, $V_{\mathrm{NT}}^{d}\left(x_{0}\right)+V_{\mathrm{NT}}^{r}\left(x_{0}\right)$.

Overall global rationality is a requirement for the agreement to arise. However, it cannot guarantee that an agreement for the joint investment project is reached and maintained until its finalization. The next section presents a mechanism which guarantees the time consistency of the cooperative solution. This property ensures the formation of a coalition to build the canal, and guarantees that no region has an incentive to deviate from the cooperative solution at any time before the completion of the canal.

\section{Time consistency of the cooperative solution}

Before we start with the analysis it is worth to define precisely what is meant and what is not meant by time consistency. In non-cooperative differential games, a solution is said to be time-consistent if players do not modify their strategies when they are allowed to recompute them at any time (along the optimal trajectory for the state variable). Or equivalently, players have no rational reason, at any further state of the game, to deviate from the policies adopted at the beginning of the game (see, Başar and Olsder (1999)). However, the definition of time consistency is different when dealing with cooperative differential games. Within this literature, time consistency addresses the problem of whether a cooperative agreement made at the start of the game, can be sustained over time. A cooperative solution is said to be time consistent if no player has an incentive to deviate from cooperation, at any point in time (the cooperative payoffs to go surpass the non-cooperative payoffs to go at any time within cooperation). That is, the property of individual rationality is satisfied at any time along the optimal cooperative trajectory for the state variable. This definition can be found, for example, in Zaccour (2008) (and references therein), and dates back to Petrosjan (1977).

After some notation and definitions of rationality, this section first proposes a sharing rule and an associated side-payment to guarantee that, overall each region is better off if the canal is built. This global side-payment, which gives rise to overall individual rationality, must be distributed along the cooperating period to guarantee that no country has an 
incentive to deviate from the cooperation at any time. Thus, an imputation distribution procedure is defined in order to guaranty time consistency. This IDP is made feasible based on an instantaneous side-payment scheme.

Next we define the instantaneous welfare for the two regions under the assumption that they behave optimally. First we consider the no-transfer scenario (subscript NT), in which no water transfer ever takes place. Then, if players agree to jointly invest to build the canal, there is a first period $[0, T)$, of cooperation (subscript $C$ ), in which both regions invest to build the canal, followed by a second period from $T$ on (no subscript), when the water transfer is available and a water market is established.

$$
\begin{array}{lll}
w_{\mathrm{NT}}^{d}=F^{d}(0,0), & w_{\mathrm{NT}}^{r}(t)=F^{r}\left(0,0, x_{\mathrm{NT}}(t), \sigma_{\mathrm{NT}}(t)\right), & \forall t \geq 0, \\
w_{\mathrm{C}}^{d}(t)=F^{d}(0,0)-C^{d}\left(I_{\mathrm{C}}^{d}(t)\right), & w_{\mathrm{C}}^{r}(t)=F^{r}\left(0,0, x_{\mathrm{C}}(t), \sigma_{\mathrm{C}}(t)\right)-C^{r}\left(I_{\mathrm{C}}^{r}(t)\right), & \forall t \in[0, T], \\
w^{d}(t)=F^{d}(p(t), \tau(t)), & w^{r}(t)=F^{r}(p(t), \tau(t), x(t), \sigma(t)), & \forall t>T,
\end{array}
$$

All control and state variables are at their corresponding optimal values. Furthermore, the price and quantity of the water market are also at their equilibrium values.

In the no-transfer scenario, the donor enjoys the constant environment amenities linked with a pristine river. Correspondingly, the recipient, with no possibility of getting water from the donor, has a strong incentive to invest in its stock of water capacity.

In contrast, if the regions agree to build the canal, within the first period of joint investment, the donor while still enjoying the full environmental amenities, has to pay the investment costs of the infrastructure. The recipient also bears the investment cost associated with the construction of the canal, while its incentive to invest in the stock of water capacity is reduced due to the expectation of future transfers. Finally, from the moment the canal is finished, the donor transfers water to the recipient at a price fixed by their dynamic interaction in the water market.

At any time $t$ within the cooperative period $[0, T]$, we define the payoffs to go when cooperating players decide to continue with cooperation, or alternatively, to suspend the agreement indefinitely. If countries maintain cooperation and finish the canal at $T$, allowing the transfer of water from this time on, under bilateral trade, the payoffs to go from this time $t \in[0, T]$ on, and for each region region $i \in\{d, r\}$ would read

$$
W^{i}(t)=\int_{t}^{T} w_{\mathrm{C}}^{i}(u) e^{-\rho(u-t)} d u+\int_{T}^{\infty} w^{i}(u) e^{-\rho(u-T)} d u,
$$

or equivalently

$$
W^{i}(t)=\int_{t}^{T} w_{\mathrm{C}}^{i}(u) e^{-\rho(u-t)} d u+V^{i}\left(x_{\mathrm{C}}(T)\right) e^{-\rho(T-t)}, \quad \forall t \in[0, T], \forall i \in\{d, r\} .
$$

Alternatively, regions might cease cooperation at this time $t$ before the canal is finished, and stick to their no-transfer strategies thereafter (no water trading ever takes place). The payoffs to go starting at this time $t$ would read

$$
W_{\mathrm{NT}}^{i}(t)=\int_{t}^{\infty} w_{\mathrm{NT}}^{i}(u ; t) e^{-\rho(u-t)} d u, \quad \forall t \in[0, T], \forall i \in\{d, r\} .
$$

Or equivalently,

$$
\begin{aligned}
& W_{\mathrm{NT}}^{i}(t)=\int_{t}^{T} w_{\mathrm{NT}}^{i}(u ; t) e^{-\rho(u-t)} d u+V_{\mathrm{NT}}^{i}\left(x_{\mathrm{NT}}(T ; t)\right) e^{-\rho(T-t)}, \quad \forall t \in[0, T], \forall i \in\{d, r\} . \\
& \text { with } w_{\mathrm{NT}}^{d}(u ; t)=w_{\mathrm{NT}}^{d} \text { constant, and } w_{\mathrm{NT}}^{r}(u ; t)=F^{r}\left(0,0, x_{\mathrm{NT}}(u ; t), s_{\mathrm{NT}}(u ; t)\right) .
\end{aligned}
$$


Assuming that the two regions have agreed to cooperate from the beginning until time $t$ prior to the completion of the canal, they will have been investing $I_{\mathrm{C}}^{d}$ and $I_{\mathrm{C}}^{r}$ in the canal and $s_{\mathrm{C}}$ in the stock of usable water capacity. Hence, this stock reached the level $x_{\mathrm{C}}(t)$. If they maintain cooperation, the optimal investment in usable water capacity would still be given by $s_{\mathrm{C}}$ and the stock would evolve to reach $x_{\mathrm{C}}(T)$ when the canal is finished. Conversely, if they halt cooperation, the optimal investment in this stock would now be driven by $s_{\mathrm{NT}}$ and the stock would evolve differently. The initial value for this stock in the second period is given by $x_{\mathrm{C}}(t)$, and for that reason the optimal path followed by this stock in this second scenario will depend on the time $t$, when cooperation ended as well as on the current time after defection $x_{\mathrm{NT}}(u ; t), u \geq t$. Obviously, at time $T$, the values $x_{\mathrm{NT}}(T ; t)$ and $x_{\mathrm{C}}(T)$ are not necessarily the same. One might expect that the expectation of a future water transfer would discourage investments in the stock of usable water capacity and hence, $x_{\mathrm{NT}}(T ; t)>x_{\mathrm{C}}(T)$.

Taking into account these definitions, at any time $t \in[0, T]$ we can define the surplus to go from cooperation from this time on for player $i$ as

$$
S^{i}(t)=W^{i}(t)-W_{\mathrm{NT}}^{i}(t), \quad \forall t \in[0, T], \forall i \in\{d, r\} .
$$

And the total surplus to go from cooperation from this time on as:

$$
S(t)=W^{d}(t)+W^{r}(t)-W_{\mathrm{NT}}^{d}(t)-W_{\mathrm{NT}}^{r}(t) \quad \forall t \in[0, T]
$$

\subsection{Overall rationality}

With the notation above, Condition 1 can be written as $W^{i}(T)>W_{\mathrm{NT}}^{i}(T)$ or $S^{i}(T)>0$ for $i \epsilon$ $\{d, r\}$; and $x_{\mathrm{C}}(T)$ in a nonempty set; and Condition 2 as $W^{d}(0)+W^{r}(0)>W_{\mathrm{NT}}^{d}(0)+W_{\mathrm{NT}}^{r}(0)$ or $S(0)>0$. Overall global rationality requires that the total investment costs of building the channel plus the environmental costs associated with the water transfer must be lower than the aggregate gains from the water transfer, which is equivalent to having a positive total surplus from cooperation. However, overall global rationality is not sufficient for cooperation, overall individual rationality must also be satisfied:

$$
W^{i}(0)>W_{\mathrm{NT}}^{i}(0) \Leftrightarrow S^{i}(0)>0, \quad \forall i \in\{r, d\} .
$$

We start by assuming that overall individual rationality is not fulfilled. From now on we make the assumption that player $i$ is worse off, while player $-i$ is better off:

$$
S^{i}(0) \equiv W^{i}(0)-W_{\mathrm{NT}}^{i}(0)<0, \quad S^{-i}(0) \equiv W^{-i}(0)-W_{\mathrm{NT}}^{-i}(0)>0 .
$$

Because we are assuming Condition 2 of overall global rationality, according to the KaldorHicks compensation criterion, in the cooperative scenario it is always possible to define a compensation payment or a side-payment from region $-i$ to region $i$, that leaves everyone as well off and at least one better off than in the scenario in which the project is not implemented. Thus, the project leads to a Pareto improvement. The more straightforward and widely used side-payment is to follow the egalitarian principle. ${ }^{14}$ Under this rule, each player attains an equal share of the global surplus from cooperation.

In our proposal, the global surplus from cooperation is shared by the two players but not necessarily in equal shares. To define each player's share, notice that as equation (12) shows,

\footnotetext{
${ }^{14}$ In this case the side-payment is defined as a payment from $-i$ to $i$ equal to half the surplus gained by $-i$ plus half the losses suffered by $i$ when the canal is jointly built.
} 
the game analyzed here is particular in the sense that cooperation leads to immediate losses (investment costs to build the canal), and it is followed by a second non-cooperative period which will determine each player's gains from the existence of the canal. Once the canal is operative, we can define the $i$-th share in the total gains which stem from the existence of the canal as

$$
\theta^{i}=\frac{V^{i}\left(x_{\mathrm{C}}(T)\right)-V_{\mathrm{NT}}^{i}\left(x_{\mathrm{C}}(T)\right)}{V^{i}\left(x_{\mathrm{C}}(T)\right)+V^{-i}\left(x_{\mathrm{C}}(T)\right)-V_{\mathrm{NT}}^{i}\left(x_{\mathrm{C}}(T)\right)-V_{\mathrm{NT}}^{-i}\left(x_{\mathrm{C}}(T)\right)}=\frac{S^{i}(T)}{S(T)}, \quad i \in\{d, r\}
$$

From Condition 1 it immediately follows that $\theta^{i} \in(0,1)$. From this definition, it is immediately obvious that $W^{i}(T)=W_{\mathrm{NT}}^{i}(T)+\theta^{i} S(T)$. This share $\theta^{i}$ is defined as the surplus for player $i$ divided by the total surplus associated with the existence of the canal. It represents player's $i$-th share of total gains, obtained in the second period and therefore it does not take into consideration the costs incurred within the cooperating period. Our proposal is that this must be his share of the total surplus from cooperation. In consequence a global side-payment from player $-i$ to player $i$ must be defined to guarantee that each player's payoffs to go under cooperation, including the side-payment, $v^{i}(0)$, equate his payoffs to go in the case of no cooperation plus the share $\theta^{i}$ of the total surplus to go at time 0 :

$$
\begin{aligned}
& v^{i}(0)=W^{i}(0)+S D=W_{\mathrm{NT}}^{i}(0)+\theta^{i} S(0), \\
& v^{-i}(0)=W^{-i}(0)-S D=W_{\mathrm{NT}}^{-i}(0)+\theta^{-i} S(0) .
\end{aligned}
$$

While $S(T)$ represents the surplus associated with the existence of the canal, $S(0)$ takes into account the gains from the existence of the canal, but also the investment costs within the first cooperative period. By construction, at time $T$ each player receives what he would have gained without the canal, plus a different share of the global surplus from the existence of the canal. According to expressions (19)-(20) this statement must be equally valid at the beginning of the cooperative agreement. Thus taking into account all investment costs to build the canal, it must still be true that each agent gets what he would have gotten without the canal plus a share from the global surplus to go, identical to the share when the canal is finished. In consequence, this proposal links players contributions to the joint investment project with the future benefits stemming from the canal.

From (19)-(20) the global side-payment from player $-i$ to player $^{15} i$ immediately follows:

$$
S D^{i}=\theta^{i}\left[W^{-i}(0)-W_{\mathrm{NT}}^{-i}(0)\right]+\theta^{-i}\left[W_{\mathrm{NT}}^{i}(0)-W^{i}(0)\right], \quad i \in\{r, d\} .
$$

This side-payment can be defined as the addition of the $i$-th share of the total gains of region $-i$ plus the $-i$-th share of the losses of region $i .{ }^{16}$

Corollary 1 By labeling the donor as $i$ and the recipient as $-i$, the global side-payment from recipient to donor would read

$$
S D^{d}=\theta^{d}\left[W^{r}(0)-W_{N T}^{r}(0)\right]+\theta^{r}\left[W_{N T}^{d}(0)-W^{d}(0)\right]=\theta^{d} S^{r}(0)-\theta^{r} S^{d}(0) .
$$

\footnotetext{
${ }^{15}$ We have assumed $S^{i}(0)<0$ and $S^{-i}(0)>0$, and hence the global side-payment goes from player $-i$ (who is better off with the cooperative agreement) to player $i$ (who is worse off). The same analysis is valid if instead $S^{i}(0)>0$ and $S^{-i}(0)<0$.

${ }^{16}$ Alternatively, the global side-payment can be interpreted as the $i$-th share of the global surplus from cooperation minus the $-i$-th gains:
}

$$
S D^{i}=\theta^{i} S^{-i}(0)-\theta^{-i} S^{i}(0)=\theta^{i} S(0)-S^{-i}(0), \quad i \in\{r, d\} .
$$


If the recipient had losses and the donor gains, then SD would be negative, and would represent a side-payment from donor to recipient.

This subsection has proposed a rule to share the global surplus from cooperation and the associated side-payment guaranteeing overall individual rationality. However, in a dynamic context, overall individual rationality is not sufficient for cooperation. Furthermore, one must prove that the cooperative solution is time consistent. The following subsection extends the sharing rule, $\theta^{i}$, to decompose the total cooperative payoff between regions and over the time interval $[0, T]$ in such a way as to guarantee time consistency. We also present an instantaneous side-payment scheme which makes this distribution possible.

\subsection{Time consistency}

The cooperative solution is time consistent if individual rationality is satisfied in every subgame starting at any time during the cooperative solution.

Definition 1 The cooperative solution without side-payments would be time consistent if and only if

$$
S^{i}(t) \equiv W^{i}(t)-W_{N T}^{i}(t) \geq 0, \quad \forall t \in[0, T], \forall i \in\{d, r\} .
$$

This is clearly not the case, since in (17) we are assuming that one of the players is initially worse off under the cooperation. However, even if individual overall rationality was satisfied by both players, time consistency would not be guaranteed. More conditions are needed.

The Kaldor-Hicks efficiency assumption in Condition 2 implies $S(0)>0$. Time consistency requires a more demanding necessary condition: A non-negative surplus to go at any time $t \in[0, T)$, which will be assumed henceforth:

\section{Condition 3}

$$
S(t)=W^{d}(t)+W^{r}(t)-W_{N T}^{d}(t)-W_{N T}^{r}(t) \geq 0 \quad \forall t \in[0, T]
$$

Given the specification of the problem, investment costs are distributed along the initial cooperative period. In contrast, the benefits from the existence of the canal arise when the cooperation ends. As times runs within the interval $[0, T]$ costs are being paid while the bulk ${ }^{17}$ of the benefits are yet to come. It is then very likely ${ }^{18}$ that the surplus to go increases with time and hence $S(0)>0$ should imply $S(t)>0$ for any $t \in[0, T]$. If it is initially beneficial to build the canal, $S(0)>0$, it seems plausible that it will be beneficial at any intermediate time to continue with the construction of the canal. Therefore, Condition 3 can be regarded as either irrelevant or not much more demanding than Condition 2.

The literature on Differential Games defines an imputation distribution procedure, $\pi^{i}(t)$, $\forall t \in[0, T], \forall i \in\{d, r\}$, as the decomposition of the joint accumulated payoffs between agents and over time. For a given IDP, each player's aggregate payoffs at the starting date, $v^{i}(0)$, is composed of the stream of payoffs from 0 to $T$ as described by the IDP, plus the value function of each region at time $T$ when the canal is finished:

$$
v^{i}(0)=\int_{0}^{T} \pi^{i}(u) e^{-\rho u} d u+V^{i}\left(x_{\mathrm{C}}(T)\right) e^{-\rho T}, \quad \forall i \in\{d, r\} .
$$

\footnotetext{
${ }^{17}$ Savings from lower investments in usable water capacity associated with the expectations of future water transfers are already realized within the cooperative period.

${ }^{18}$ It has not been analytically proved, although it is shown in the numerical example.
} 
Next, we define a time-consistent IDP.

Definition 2 For a given IDP, $\pi^{i}(u)$, the payoffs to go for player $i \in\{d, r\}$ at any time $t \in[0, T]$ read: ${ }^{19}$

$$
v^{i}(t)=\int_{t}^{T} \pi^{i}(u) e^{-\rho(u-t)} d u+V^{i}\left(x_{C}(T)\right) e^{-\rho(T-t)},
$$

assuming that cooperation has lasted till $t$, and $x_{C}(T)$ is the stock of water capacity at the time when the canal becomes operative, T. This IDP is time consistent under condition:

$$
v^{i}(t)=W_{N T}^{i}(t)+\phi^{i}(t) S(t) \quad \forall t \in[0, T], \forall i \in\{d, r\},
$$

with $\phi^{i}(t)$ a differentiable function satisfying $\phi^{i}(t) \in[0,1]$ for all $t \in[0,1]$.

While the payoffs to go are defined in (24), time consistency requires that they satisfy (25). This condition states that by continuing cooperation, player $i$ 's payoffs to go equate his payoffs to go in case of defection plus a share $\phi^{i}(t)$ of the total surplus to go. Hence as long as the surplus to go remains non-negative at any time $t \in[0, T]$, as stated in Condition 3 no player would do better by deviating from cooperation and the IDP would be time consistent. An alternative way to write condition $(25)$ is

$$
\frac{v^{i}(t)-W_{\mathrm{NT}}^{i}(t)}{S(t)}=\phi^{i}(t), \quad \forall t \in[0, T], \forall i \in\{d, r\} .
$$

From this expression, $\phi^{i}(t)$ can be interpreted as a measure of the excess gain for player $i$ from any time $t \in[0, T]$ on, if cooperation is maintained with the IDP $\pi^{i}(t)$, in relative terms to the total surplus to go. Thus, it represents player's $i$ willingness to continue cooperation at time $t \in[0, T]$, under this IDP, $\pi^{i}(t)$.

Given this definition of a time-consistent IDP, the following questions are worth analyzing. First we show that such a time-consistent IDP exists and analyze its uniqueness/multiplicity. Finally, we prove that it is feasible and provides a side-payment scheme under which each player gets its share, as stated by the IDP.

The proof that there exists an imputation distribution procedure which satisfies definition (24) and condition (25) is split in two parts. First, we prove that $v^{i}(t)$ given in the definition (24) satisfies condition (25) at the final time $T$ under an additional condition on function $\phi^{i}(t)$. And second we obtain the IDP for which the time derivatives of the expressions (24) and $(25)$ are identical for any $t \in[0, T)$.

Lemma 1 If function $\phi^{i}(t)$ satisfies $\phi^{i}(T)=\theta^{i}$, (given by (18)), then expressions (24) and (25) evaluated at $T$, both lead to $v^{i}(T)=V^{i}\left(x_{C}(T)\right)$.

Proof. See Appendix

Proposition 1 The expression of $\dot{v}^{i}(t)$ for player $i \in\{d, r\}$ at any time $t \in[0, T)$, obtained as the time derivative of the definition (24) or of the expression in condition (25) are identical under the IDP:

$$
\pi^{i}(t)=w_{N T}^{i}(t)+\phi^{i}(t) s(t)-\left(\phi^{i}\right)^{\prime}(t) S(t)+\phi^{i}(t) \Theta^{-i}(t)-\phi^{-i}(t) \Theta^{i}(t),
$$

with $s(t)=w_{C}^{i}(t)+w_{C}^{-i}(t)-w_{N T}^{i}(t)-w_{N T}^{-i}(t)$, the instantaneous surplus from cooperation at timet (typically negative), and $\Theta^{i}(t)=\int_{t}^{T} \dot{w}_{N T}^{i}(u ; t) e^{-\rho(u-t)} d u+\left(V_{N T}^{i}\right)_{x_{N T}}^{\prime} \dot{x}_{N T}(T ; t) e^{-\rho(T-t)}$.

${ }^{19}$ See, for example, Zaccour (2008). 
Proof. See Appendix

Remark 1 In consequence, for any differentiable function $\phi^{i}(t)$ taking values between 0 and 1 , and satisfying $\phi^{i}(T)=\theta^{i}$, where $\theta^{i}$ is given by equation (18), a time-consistent IDP exists.

In what follows, for the sake of clarity we focus on the particular case of a constant $\phi^{i}(t)$. Under this assumption the following corollary is obvious.

Corollary 2 Under the assumption of a constant $\phi^{i}(t)$, the time-consistent IDP is unique and it is obtained if and only if ${ }^{20} \phi^{i}(t)=\phi^{i}=\theta^{i}$ for all $t \in[0,1]$ :

$$
\pi^{i}(t)=w_{N T}^{i}(t)+\theta^{i} s(t)+\theta^{i} \Theta^{-i}(t)-\theta^{-i} \Theta^{i}(t), \quad \forall t \in[0, T), i \in\{r, d\} .
$$

Note that for this case of $\phi^{i}(t)=\theta^{i}$, condition (26) states that at any point in time within cooperation, what player $i$ has to win by maintaining cooperation (in relative terms to the total surplus from cooperation) remains constant. And this constant must be identical to the relative value that this player assigns to the existence of the canal, as defined in (18). Note that alternatively, any non-constant function $\phi^{i}(t)$ taking values within the interval $[0,1]$ verifying that the time derivatives of (24) and (25) are the same would give rise to a timeconsistent IDP, as long as this function satisfies $\lim _{t \rightarrow T} \phi^{i}(t)=\theta^{i}$. This could be interpreted as player's $i$ willingness to cooperate converging to the value, $\theta^{i}$, that he assigns to the existence of the canal at time $T$.

Corollary 3 By labeling the donor as $i$ and the recipient as $-i$, the IDP can be written as

$$
\begin{array}{ll}
\pi^{d}(t)=w_{N T}^{d}(t)+\theta^{d} s(t)+\theta^{d} \Theta^{r}(t), & \forall t \in[0, T), \\
\pi^{r}(t)=w_{N T}^{r}(t)+\theta^{r} s(t)-\theta^{d} \Theta^{r}(t), & \forall t \in[0, T) .
\end{array}
$$

For the recipient $\Theta^{r}(t)$ represents the effect of a marginal delay in defection (or a longer cooperation), on the path of investment in usable water capacity and, in consequence, on the evolution of the stock of usable water capacity and hence, on the flow of instantaneous welfare of the recipient from this time $t$ (of defection) on. Hence, it measures how the payoffs to go for a deviating recipient (net of discount) are affected if cooperation is maintained a little longer. ${ }^{21}$ From the point of view of the recipient, a longer cooperation has an unclear effect on his future after-defection payoffs. On the one hand, the costs of the investment in usable water capacity are shared with the donor for a little longer, reducing future investments costs. However, at the same time, since the reaction in the form of higher investments in usable water capacity is delayed, this stock adjusts less rapidly, so negatively affecting after-defection payoffs.

As for the donor, since he has no decision to make after the moment $t$ when cooperation stops, and since his after-defection payoffs are not dependent on the usable water capacity, logically, there will be no marginal effects associated with delays in the time of defection, $\Theta^{d}(t)=0, \forall t \in[0, T)$.

\footnotetext{
${ }^{20}$ Proof is straightforward since any other constant value for $\phi^{i}(t)$ would not satisfy Lemma 1.

${ }^{21}$ The complete effect of a marginal delay in defection on the payoffs to go for region $i$ has two other components as shown in (33). The payoffs to go are reduced in the instantaneous payoff corresponding to time $t,-w_{\mathrm{NT}}^{i}(t)$, (in fact they are increased because the instantaneous payoff is typically negative). Secondly, the initial time $t$ moves forward, payoffs to go are valued later and then the value increases by $\rho W_{\mathrm{NT}}^{i}(t)$.
} 
We have proved the existence of a time-consistent IDP. We have also shown that the IDP is unique if a constant $\phi^{i}(t)$ is sought. In contrast, infinitely more other IDPs would also be time-consistent as long as $\phi^{i}(t)$ converges to $\theta^{i}$ as $t$ approaches $T$. As a final step we will elaborate on the feasibility of these IDPs. First, we prove that the IDP computed in (27) is feasible. And second, we define the instantaneous side-payment that makes the IDP possible.

Lemma 2 At any time $t \in[0, T]$, the instantaneous payoffs provided by the IDP for the two players matches the instantaneous joint cooperative payoff: $\pi^{i}(t)+\pi^{-i}(t)=w_{C}^{i}(t)+w_{C}^{-i}(t)$.

Proof is straightforward from the definition of the IDP in (27).

An immediate consequence from this Lemma is that an instantaneous or continuous sidepayment from country $-i$ to country $i, s d(t)$, can be defined from 0 to $T$ in such a way as to guarantee that each country gets the instantaneous payoff defined by the IDP in (27).

Proposition 2 The instantaneous side-payment from player $-i$ to player $i$ is given by

$$
s d(t)=\theta^{i}\left[w_{C}^{-i}(t)-w_{N T}^{-i}(t)\right]+\theta^{-i}\left[w_{N T}^{i}(t)-w_{C}^{i}(t)\right]+\theta^{i} \Theta^{-i}(t)-\theta^{-i} \Theta^{i}(t)
$$

satisfies that $\pi^{i}(t)=w_{C}^{i}(t)+s d(t)$ and $\pi^{-i}(t)=w_{C}^{-i}(t)-s d(t)$ for all $t \in[0, T)$.

Corollary 4 Labeling the donor with $i$ and the recipient with -i, the instantaneous sidepayment from recipient to donor can be written as

$$
s d(t)=\theta^{d}\left[w_{C}^{r}(t)-w_{N T}^{r}(t)\right]+\theta^{r}\left[w_{N T}^{d}(t)-w_{C}^{d}(t)\right]+\theta^{d} \Theta^{r}(t), \quad \forall t \in[0, T) .
$$

A negative value of sd(t) would represent a side-payment from donor to recipient.

We can distinguish two parts in the instantaneous side-payment in (31). The first part is the addition of the donor's share ${ }^{22} \theta^{d}$, of the recipient's instantaneous gains from cooperation (typically negative due to the costs of building the canal) and the recipient's share, $\theta^{r}$, of the donor's instantaneous saving if they do not cooperate in building the canal (typically positive). This part corresponds to the instantaneous version of the global side-payment, $S D$, in (22), the only difference being that, in overall terms, the construction of the canal and its ulterior utilization are assumed to be beneficial for the recipient and detrimental for the donor; however, in instantaneous terms, both countries incur costs throughout the cooperative period when the canal is being built. Therefore, the instantaneous payoffs under cooperation are typically lower than under no transfer.

The second part of this instantaneous side-payment is given by the donor's share of the marginal effect of a delay in defection on the payoffs to go for the recipient (if cooperation ends before the construction of the canal), $\phi^{d} \Theta^{r}(t)$.

Remark 2 An alternative expression for the instantaneous side-payment in (31) would be $s d(t)=\theta^{d}\left[\dot{W}_{N T}^{r}(t)-\rho W_{N T}^{r}(t)-\left(\dot{W}^{r}(t)-\rho W^{r}(t)\right)\right]+\theta^{r}\left[\dot{W}^{d}(t)-\rho W^{d}(t)-\left(\dot{W}_{N T}^{d}(t)-\rho W_{N T}^{d}(t)\right)\right]$.

\footnotetext{
${ }^{22}$ Constant $\theta^{d}$ (resp. $\theta^{r}$ ) represents the donor's share (resp. the recipient's share) of the total gains from the existence of the canal. We will refer to player's share for brevity.
} 
Note that $\dot{W}_{\mathrm{NT}}^{i}(t)-\rho W_{\mathrm{NT}}^{i}(t)$ and $\dot{W}^{i}(t)-\rho W^{i}(t)$ represent the temporal evolution of the payoffs to go (net of discount) under either the no-transfer solution or the cooperative scenario, at time $t \in[0, T)$. Thus, the first term in brackets in (32) represents the gap in the temporal evolution of the recipient's payoffs to go under no transfer and cooperation. That is how much faster the recipient's payoffs to go increase under the no-transfer case than under cooperation. In fact, one might expect that the payoffs to go would decrease under the no-transfer solution and increase under cooperation. Hence, this term would likely be negative. Correspondingly, the second term in brackets shows how much faster the donor's payoffs to go are increased in the cooperative scenario than in the no-transfer solution (a likely positive term).

Remark 3 We have described the water market as a bilateral monopoly, following Cabo et al. (2014). This is one particular specification, although one might also think of a water market with one region having monopolistic power. Thus defining a game played a la Stackelberg with either the donor or the recipient taking the role of the leader. Alternatively, more complex specifications involving interactions between the donor's decision on the water transfer and the dynamics of the usable water capacity could also be considered. Regardless of the water market specification, the time consistent IDP derived in this Section 3 would be equally valid as long as Condition 1 is satisfied.

\section{Application: specific functional forms}

This section computes the IDP described in the previous section for the water transfer example. Specific functional forms are assumed in order to attain analytical solutions. The dynamic of the usable water capacity in (8) is linear. Therefore, to have an analytically tractable problem we consider here quadratic instantaneous profit functions, as in Cabo et al. (2014). For the donor:

$$
F^{d}(p, \tau)=E^{d}(\tau)+p \tau=c\left(R-\frac{\tau^{2}}{2 R}\right)+p \tau, \quad c>0,
$$

with $R>0$ being the constant water surplus in the donor's river basin. In the absence of water transfer, environmental amenities increase linearly with the water surplus: $c R$. Furthermore, we consider that the marginal reduction in environmental amenities is inversely proportional to this surplus, while proportional to the water transferred: $c \tau(t) / R$.

For the recipient, output increases at a decreasing rate with the total quantity of water (either the usable water capacity or the transfer). This is represented by a linear-quadratic function in $\tau+x$. We also consider quadratic costs of investment in usable water capacity.

$$
F^{r}(p, \tau, x, \sigma)=d\left(\tau+x-\alpha \frac{(\tau+x)^{2}}{2}\right)-p \tau-\beta \frac{\sigma^{2}}{2} \quad d, \alpha, \beta>0 .
$$

For illustration purposes, we consider the parameters' values ${ }^{23}$ which guarantee conditions 1 , 2 and 3: both players are better off if a canal exists, the project to build the canal improves overall global welfare and, moreover, the surplus to go from cooperation is positive at any time within cooperation. The equilibrium for the non-cooperative game can be found in

\footnotetext{
${ }^{23} c=4.7084, d=2.1275, \rho=0.001, \delta=0.1, R=593.67, \alpha=0.0135$, as in Cabo et al. (2014). And $x(0)=0, z^{d}=z^{r}=10, K_{0}=0, \bar{K}=100$.
} 
Cabo et al. (2014), and the cooperative solution is presented in the Appendix. For the chosen parameters we obtain:

$T^{*}=30.76, x_{\mathrm{C}}^{T}=47.97, \theta^{d}=0.344, \theta^{r}=0.656, S(0)=V_{\mathrm{C}}\left(x_{0}\right)-\left(V_{\mathrm{NT}}^{d}\left(x_{0}\right)+V_{\mathrm{NT}}^{r}\left(x_{0}\right)\right)=1972.79$.

However, although we assume overall global rationality, we also consider that cooperation increases the recipient's accumulated welfare, $S^{r}(0)=V_{\mathrm{C}}^{r}\left(x_{0}\right)-V_{\mathrm{NT}}^{r}\left(x_{0}\right)=2048.06$, but decreases the donor's accumulated welfare $S^{d}(0)=V_{\mathrm{C}}^{d}\left(x_{0}\right)-V_{\mathrm{NT}}^{d}\left(x_{0}\right)=-75.26$. In consequence, without side-payment, the latter would not agree to cooperate to build the canal.

The surplus to go under cooperation is initially positive, $S(0)=1972.79$, and increases with time from 0 to $T$. Therefore, Condition 2 and Condition 3 are satisfied.
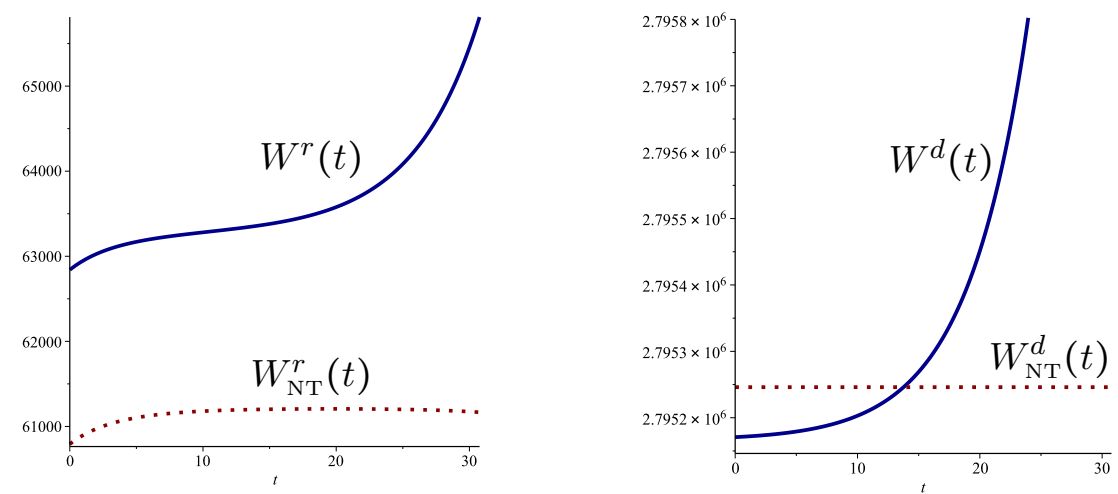

Figure 1: Payoff to go at $t$, under cooperation vs. no transfer.

Figure 1 displays the payoffs to go if the countries accept cooperation, $W^{i}$, and under the no-transfer scenario (when no canal is ever built) $W_{N T}^{i}$. According to this figure, at the beginning of the cooperative period, the donor's payoffs to go would be higher if he does not cooperate and sticks to his strategy in the no-transfer scenario. It would not be rational for the donor to maintain cooperation. Thus, the cooperative solution is not time consistent, and would never be implemented unless an adequate mechanism gives the donor an incentive to cooperate.
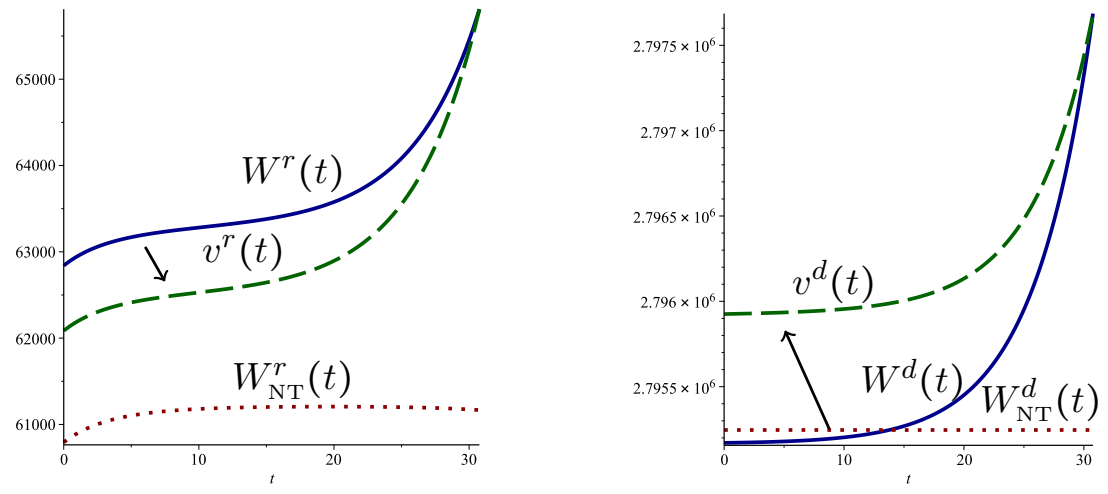

Figure 2: Payoff to go after side-payment at $t$, under cooperation vs. no transfer.

Figure 2 shows how the payoffs to go are modified if the instantaneous side-payment in (30) is implemented. The payoffs to go after the side-payment, $v^{i}(t)$, correspond to 
the IDP defined in (27). These are greater than their respective payoffs to go under the alternative no-transfer scenario in case of defection. Therefore, both cooperating players are individually rational at any time and this IDP is time consistent. Countries will stick to their cooperative investment strategies and the canal will be finished.

\section{Conclusions}

This paper studies the dynamic interaction between two countries with differences in precipitation rates and water productivity. Water inflows in the river basin located in the country labeled as the donor are relatively higher than in the recipient country. After satisfying the local demand the donor still enjoys a flow of water surplus. Although this surplus is not used in the productive process, it is valuable because it helps to preserve the environmental amenities provided by the river. In the recipient economy water is highly productive (one can think of different uses like irrigation, industry, municipal water supply, etc.), but the river basin in this country suffers from a chronic shortage of water. In a subsidiary way, this country has the possibility to invest in alternative water supplies, like desalination plants, water savings or water recycling. All in all, if the donor's river basin could transfer part of its water surplus to the recipient's, overall productivity would be increased. Thus, the existence of a canal allowing this water transfer would create a water market, so increasing overall productivity. The recipient would be willing to pay a price for the water its economy demands. Correspondingly, the donor would be willing to accept a price for the excess water transferred, as long as this price offsets the environmental losses associated with the transfer. Therefore, if the productivity of water in the recipient's economy is sufficiently high with respect to the losses in the environmental amenities provided by the river in the donor's country, then the existence of the canal, and the water market it makes possible, may signify a gain for both regions. Nonetheless, there is no reason for these gains to be necessarily the same for both players.

The previous situation is an example of an infrastructure which improves the welfare of two players in a non-cooperative differential game, although not necessarily in the same way. The question addressed in this paper has been: under which conditions would they agree to a first period of cooperation in a joint investment project to build this infrastructure? To analyze this question, we define a two-regime differential game between the two countries. In a second period, when the canal exists, a non-cooperative differential game of infinite duration describes the water market they are engaged in. However, for the canal to exist, they must agree to cooperate in a first period to jointly build the canal.

This is an example of a cooperative game in which cooperation does not imply an immediate reward but a burden: the investment costs of building the canal. The asymmetric benefits from the cooperation only come at the end of the game, when the cooperation stops, and they are determined in a subsequent non-cooperative differential game describing the water market made possible by the canal. Therefore, the standard question in cooperative game theory on how to share the gains from cooperation is changed to a new question: how to share the burden from cooperation taking into account future uneven gains? This sharing rule must guarantee that the canal is actually finished. That is, it must be defined in such a way that the cooperative solution satisfies the property of time consistency.

We assume first that the joint investment project is profitable. This means a positive 
joint aggregate surplus from cooperation. Further, we assume a positive surplus to go initially and at any time within the cooperative period. Lastly, if the canal is built, both players are better off with the existence of the canal. In overall terms for the whole time period we propose a sharing rule. At the initial time (when they start building the canal) this rule is based on the principle that each player's share of the total gains stemming from the existence of the canal (which do not include the costs of building the infrastructure) must also define this player's share of the total surplus from cooperation. This sharing rule assigns each country its overall gains in the case of no transfer, plus his share of the global surplus from cooperation. A global side-payment is defined to guarantee that this share is attained.

This basic principle is extended from overall to instantaneous terms, rendering the cooperative agreement time consistent. The joint aggregate payoffs under cooperation is distributed between players and over time by an imputation distribution procedure. The IDP is defined to satisfy a dynamic version of the basic principle previously stated for aggregate payoffs. At every time within cooperation, each player's share of the surplus to go (including pending costs and future benefits) must be equal or must converge to his share in the total benefits associated with the existence of the canal. Then, at any time within cooperation each country's payoffs to go equate what it would get with no transfer plus the previously-proposed share of the global surplus to go. In consequence, the IDP is time consistent and no country has an incentive to deviate from cooperation. For this sharing rule we are able to characterize the instantaneous payoffs which define the IDP for each country. More importantly, the time-consistent IDP is feasible. At any time within cooperation an instantaneous side-payment guarantees that each player gets what is specified by the IDP. This side-payment scheme is analytically established in the paper.

The central idea that each player's share of the surplus to go at any time within cooperation has to be equal or converge towards this player's share of the future benefits associated with the canal, guarantees the basic principle of contributing according with future benefits.

Although we have presented this idea for the water transfer example, it can be extended to economic situations in which two players cooperate in a joint research project which leaves the two agents in a better position from the moment when the research objective is achieved on. An example could be a joint research project implemented by to firms looking for a new product or technology which improves their revenues from the moment when the product is placed on the market or the technology is put into practice. Similar characteristics are also present in transboundary or global environmental problems. Cooperating countries bear current costs (of progressive emissions reductions) and obtain future (uncertain and often uneven) benefits from a cleaner environment. We consider this an interesting line for future research, with additional difficulties, like non-existence of markets to capture the benefits from cooperation or free riding problems.

\section{Acknowledgements}

We would like to especially thank Katrin Erdlenbruch for her valuable comments. We are also grateful to M. Finus, G. Martín-Herrán, G. Zaccour and participants in the seminars given in UAM (Madrid, Spain, September 2015), GERAD (Montreal, Canada, October 2015) and LAMETA (Montpellier, France, February 2016). 
Francisco Cabo has been partially supported by MEC under project ECO2014-52343-P, cofinanced by FEDER funds. The authors acknowledge the support by COST Action IS1104 "The EU in the new economic complex geography: models, tools and policy evaluation".

\section{References}

[1] Ambec, S. and Ehlers, L. (2008) "Sharing a river among satiable agents." Games and Economic Behavior, 64, pp. 35-50

[2] Ambec, S. and Sprumont, Y. (2002) "Sharing a river." Journal of Economic Theory, 107, pp. 453-462

[3] Başar, T. and Olsder, G.J. (1999), Dynamic Noncooperative Game Theory, 2nd edition, Classics in Applied Mathematics, SIAM, Philadelphia.

[4] Ballestero, E. (2004) "Inter-Basin water transfer agreements: A decision approach to quantity and price." Water Resources Management, 18, pp. 75-88.

[5] Bhaduri, A. and Barbier, E.B. (2008) "International water transfer and sharing: The case of the Ganges river." Environment and Development Economics, 13, ppp. 29-51.

[6] Cabo, F., Erdlenbruch, K. and Tidball, M (2014) "Dynamic management of water transfer between two interconnected river basins." Resource and Energy Economics, 37, pp. 17-38.

[7] Che, Y-K. and Sákovics, J. (2004) "A dynamic theory of holdup," Econometrica, 72, 1063-1103.

[8] Ehtamo, H. and Hämaläinen, R.P. (1986) "On affine incentives for dynamic decision problems." in Dynamic Games and Applications in Economics, T. Basar (ed.), Springer, Berlin, pp. 47-63.

[9] Ehtamo, H. and Hämaläinen, R.P. (1989) "Incentive strategies and equilibria for dynamic games with delayed information," Journal of Optimization Theory and Applications, 63, pp. 355-370.

[10] Ehtamo, H. and Hämaläinen, R.P. (1993) "A Cooperative incentive equilibrium for a resource management problem," Journal of Economic Dynamics and Control, 17, pp. 659-678.

[11] Jørgensen, S. and Zaccour, G. (2001) "Time consistent side payments in a dynamic game of downstream pollution," Journal of Economic Dynamics and Control, 25, 19731987.

[12] Kilgour, M. and Dinar, A. (1995) "Are stable agreements for sharing international river waters now possible?," Policy Research Working Paper Series 1474, The World Bank.

[13] Kilgour, M. and Dinar, A. (2001) "Flexible Water Sharing within an International River Basin," Environmental and Resource Economics, 18, pp. 43-60.

[14] Kort, P. M. and Navas, J. (2007) "Time to complete and research joint ventures: A differential game approach," Journal of Economic Dynamics and Control, 31, pp. 16721696.

[15] Lopes, P.D. (2008) "Water with borders: the institutional postponement of international water trade." International Studies Association Annual Convention, San Francisco. 
[16] Petrosjan, L. (1977) "Stable solutions of differential games with many participants." Viestnik of Leningrad University, 19, pp. 46-52.

[17] Petrosjan, L. (1997) "Agreeable solutions in differential games." International Journal of Mathematics, Game Theory and Algebra. 7, pp. 165-177.

[18] Rincón-Zapatero, J.P., Martín-Herrán, G. and Martinez, J. (2000) "Identification of efficient Subgame-perfect Nash Equilibria in a class of Differential Games." Journal of Optimization Theory and Applications, 104, pp. 235-242.

[19] Zaccour, G. (2008) "Time consistency in cooperative differential games: A tutorial." INFOR Information Systems and Operational Research, 46, pp. 81-92. 


\section{Appendix}

\section{Proof of Lemma 1}

From (24) it is immediately obvious. From (25) and the assumption $\phi^{i}(T)=\theta^{i}$ :

$v^{i}(T)=W_{\mathrm{NT}}^{i}(T)+\phi^{i}(T) S(T)=V_{\mathrm{NT}}^{i}\left(x_{\mathrm{C}}(T)\right)+\frac{V^{i}\left(x_{\mathrm{C}}(T)\right)-V_{\mathrm{NT}}^{i}\left(x_{\mathrm{C}}(T)\right)}{S(T)} S(T)=V^{i}\left(x_{\mathrm{C}}(T)\right)$, for all $i \in\{d, r\}$.

\section{Proof of Proposition 1}

Computing the time derivatives in expressions (12) and (13) we get:

$$
\begin{aligned}
& \dot{W}^{i}(t)=-w_{\mathrm{C}}^{i}(t)+\rho W^{i}(t), \\
& \dot{W}_{\mathrm{NT}}^{i}(t)=-w_{\mathrm{NT}}^{i}(t)+\rho W_{\mathrm{NT}}^{i}(t)+\int_{t}^{T} \dot{w}_{\mathrm{NT}}^{i}(u ; t) e^{-\rho(u-t)} d u+\left(V_{\mathrm{NT}}^{i}\right)_{x_{\mathrm{NT}}}^{\prime} \dot{x}_{\mathrm{NT}}(T ; t) e^{-\rho(T-t)} .
\end{aligned}
$$

And hence,

$$
\begin{aligned}
& \dot{S}(t)=-s(t)+\rho S(t)-\left[\left(V_{\mathrm{NT}}^{i}\right)_{x_{\mathrm{NT}}}^{\prime}+\left(V_{\mathrm{NT}}^{-i}\right)_{x_{\mathrm{NT}}}^{\prime}\right] \dot{x}_{\mathrm{NT}}(T ; t) e^{-\rho(T-t)} \\
& -\int_{t}^{T}\left(\dot{w}_{\mathrm{NT}}^{i}(u ; t)+\dot{w}_{\mathrm{NT}}^{-i}(u ; t)\right) e^{-\rho(u-t)} d u .
\end{aligned}
$$

Taking this into account, and since $\phi^{i}(t)$ is differentiable, the time derivatives of expressions (24) and (25) read

$$
\begin{aligned}
& \dot{v}^{i}(t)=-\pi^{i}(t)+\rho v^{i}(t), \\
& \dot{v}^{i}(t)=-w_{\mathrm{NT}}^{i}(t)+\rho v^{i}(t)-\phi^{i}(t) s(t)+\left[\phi^{-i}(t)\left(V_{\mathrm{NT}}^{i}\right)_{x_{\mathrm{NT}}}^{\prime}-\phi^{i}(t)\left(V_{\mathrm{NT}}^{-i}\right)_{x_{\mathrm{NT}}}^{\prime}\right] \dot{x}_{\mathrm{NT}}(T ; t) e^{-\rho(T-t)} \\
& -\phi^{i}(t) \int_{t}^{T} \dot{w}_{\mathrm{NT}}^{-i}(u ; t) e^{-\rho(u-t)} d u+\phi^{-i}(t) \int_{t}^{T} \dot{w}_{\mathrm{NT}}^{i}(u ; t) e^{-\rho(u-t)} d u+\left(\phi^{i}\right)^{\prime}(t) S(t) .
\end{aligned}
$$

And these two expressions equate for the IDP in (27)

\section{Solving a LQ game when cooperation is maintained}

To find a solution to the problem described in (7), (8) and (9) we define the Hamiltonian: $H\left(x, K, \sigma, I^{r}, I^{d}, \lambda, \mu\right)=F^{d}(0,0)+F^{r}(0,0, x, \sigma)-C^{d}\left(I^{d}\right)-C^{r}\left(I^{r}\right)+\lambda(s-\delta x)+\mu\left(I^{d}+I^{r}\right)$.

Assuming quadratic costs, $\frac{z^{i}}{2}\left(I^{i}\right)^{2}, i \in\{d, r\}$, first order conditions give ${ }^{24}$

$$
\begin{gathered}
\lambda=\beta \sigma, \quad \mu=z^{d} I^{d}=z^{r} I^{r}, \\
\dot{K}=\frac{z^{d}+z^{r}}{z^{d} z^{r}} \mu, \\
\dot{\mu}=\rho \mu, \\
\dot{x}=\frac{\lambda}{\beta}-\delta x, \\
\dot{\lambda}=(\rho+\delta) \lambda-d(1-\alpha x), \\
H\left(x(T), K(T), \sigma(T),\left(I^{r}\right)(T),\left(I^{d}\right)(T), \lambda(T), \mu(T)\right)-\rho\left[V^{d}(x(T))+V^{r}(x(T))\right]=0 .
\end{gathered}
$$

\footnotetext{
${ }^{24}$ Similarly, one might consider lineal quadratic investment costs.
} 
The system of differential equations in $x, \lambda$

$$
\left(\begin{array}{c}
\dot{x} \\
\dot{\lambda}
\end{array}\right)=\left(\begin{array}{cc}
-\delta & 1 / \beta \\
\alpha d & \rho+\delta
\end{array}\right)\left(\begin{array}{c}
x \\
\lambda
\end{array}\right)+\left(\begin{array}{c}
0 \\
-d
\end{array}\right),
$$

can be solved separately, obtaining

$$
\left(\begin{array}{c}
x_{\mathrm{C}}(t) \\
\lambda_{\mathrm{C}}(t)
\end{array}\right)=C_{1}\left(\begin{array}{c}
\xi_{x}^{1} \\
\xi_{\lambda}^{1}
\end{array}\right) e^{r_{1} t}+C_{2}\left(\begin{array}{c}
\xi_{x}^{2} \\
\xi_{\lambda}^{2}
\end{array}\right) e^{r_{2} t}+\left(\begin{array}{c}
x_{p} \\
\lambda_{p}
\end{array}\right)
$$

where

$$
x_{p}=\frac{d}{\alpha d+\beta \delta(\rho+\delta)}, \quad \lambda_{p}=\frac{d \beta \delta}{\alpha d+\beta \delta(\rho+\delta)}
$$

and $\bar{\xi}^{1}=\left(\xi_{x}^{1}, \xi_{\lambda}^{1}\right)$ and $\bar{\xi}^{2}=\left(\xi_{x}^{2}, \xi_{\lambda}^{2}\right)$ are the eigenvectors associated to eigenvalues:

$$
r_{1}=\frac{\beta \rho-\sqrt{\beta(\rho+\delta)^{2}+4 \alpha d \beta}}{2}<0, \quad r_{2}=\frac{\beta \rho+\sqrt{\beta(\rho+\delta)^{2}+4 \alpha d \beta}}{2}>0 .
$$

Together with equation (36) conditions for this problem are: $x(0)=x_{0}, \lambda(T)=\frac{\partial S C}{\partial x}(x(T))$. Taking into account a lineal-quadratic differential game defining the water market after the finalization of the canal, as described in Cabo et al. (2014), the value functions for donor and recipient will be second order polynomials: $V^{i}(x)=a^{i} x^{2} / 2+b^{i} x+c^{i}, \forall i \in\{d, r\}$. Therefore, $(\partial S C / \partial x)(x(T))=\partial\left[V^{d}(x(T))+V^{r}(x(T))\right] / \partial x$ can be written as $a x(T)+b$, where $a=a^{d}+a^{r}$ and $b=b^{d}+b^{d}$, then these two conditions can be written as

$$
\begin{aligned}
& C_{1} \xi_{x}^{1}+C_{2} \xi_{x}^{2}=x_{0}-x_{p} \\
& C_{1}\left(\xi_{\lambda}^{1}-a \xi_{x}^{1}\right) e^{r_{1} T}+C_{2}\left(\xi_{\lambda}^{2}-a \xi_{x}^{2}\right) e^{r_{2} T}=a x_{p}-\lambda_{p}+b
\end{aligned}
$$

and the constants can be computed as functions of $T: C_{1 \mathrm{C}}(T)$ and $C_{2 \mathrm{C}}(T)$. For any $t \in[0, T]$, the optimal paths for $x$ and $\lambda$ would be

$$
\begin{aligned}
& x_{\mathrm{C}}(t)=C_{1 \mathrm{C}}(T) \xi_{x}^{1} e^{r_{1} t}+C_{2 \mathrm{C}}(T) \xi_{x}^{2} e^{r_{2} t}+x_{p} \\
& \lambda_{\mathrm{C}}(t)=C_{1 \mathrm{C}}(T) \xi_{\lambda}^{1} e^{r_{1} t}+C_{2 \mathrm{C}}(T) \xi_{\lambda}^{2} e^{r_{2} t}+\lambda_{p}
\end{aligned}
$$

Similarly, we can compute the optimal capital stock and its costate:

$$
K_{\mathrm{C}}(t)=\frac{\bar{K}-k_{0}}{e^{\rho T}-1} e^{\rho t}+\frac{k_{0} e^{\rho T}-\bar{K}}{e^{\rho T}-1}, \quad \mu_{\mathrm{C}}(t)=\frac{z^{d} z^{r}}{z^{d}+z^{r}} \frac{\left(\bar{K}-k_{0}\right) \rho}{e^{\rho T}-1} e^{\rho t} .
$$

And the optimal time $T$ at which the canal must be finished given by equation (36)

\section{Solving a LQ game with defection at time $t$}

We analyze the no water transfer scenario starting at time $t \in[0, T)$ and assuming cooperation up until this point. This is the standard problem but starting at $\left(t, x_{\mathrm{C}}(t)\right)$. The donor faces no optimization problem, while the recipient solves the problem:

$$
\max _{\sigma} \int_{t}^{\infty}\left(d\left(x-\frac{\alpha}{2} x^{2}\right)-\frac{\beta}{2} \sigma^{2}\right) e^{-\rho u} d u, \quad \dot{x}=\sigma-\delta x, \quad x(t)=x_{\mathrm{C}}(t) .
$$

The dynamics of the state and co-state variables are identical to those in the cooperative problem, stated in (37), whose solution reads

$$
\left(\begin{array}{l}
x(u) \\
\lambda(u)
\end{array}\right)=C_{1}\left(\begin{array}{c}
\xi_{x}^{1} \\
\xi_{\lambda}^{1}
\end{array}\right) e^{r_{1}(u-t)}+C_{2}\left(\begin{array}{c}
\xi_{x}^{2} \\
\xi_{\lambda}^{2}
\end{array}\right) e^{r_{2}(u-t)}+\left(\begin{array}{c}
x_{p} \\
\lambda_{p}
\end{array}\right), \quad u>t .
$$


where $x_{p}, \lambda_{p}, r_{1}, r_{2}, \bar{\xi}^{1}$ and $\bar{\xi}^{2}$, are identical to those already obtained in the cooperative case. The transversality condition $\lim _{u \rightarrow \infty} \lambda(u) x(u) e^{-\rho(u-t)}=0$, requires $C_{2}=0$. This together with condition $x(t)=x_{\mathrm{C}}(t)$ gives $C_{1}=\left(x_{\mathrm{C}}(t)-x_{p}\right) / \xi_{x}^{1}$ and the solutions of $x$ and $\lambda$ for any time $u$ after $t$ when cooperation halts:

$$
\begin{gathered}
x_{\mathrm{NT}}(u ; t)=\left(x_{\mathrm{C}}(t)-x_{p}\right) e^{r_{1}(u-t)}+x_{p}, \\
\lambda_{\mathrm{NT}}(u ; t)=\left(x_{\mathrm{C}}(t)-x_{p}\right) \frac{\xi_{\lambda}^{1}}{\xi_{x}^{1}} e^{r_{1}(u-t)}+\lambda_{p} .
\end{gathered}
$$

We can now compute the derivative of $x_{\mathrm{NT}}(T ; t)$ with respect to the time $t$ when cooperation ceases, used in the definition of $\Theta^{i}(t)$ in Proposition 1.

$$
\begin{aligned}
& \frac{d}{d t}\left(x_{\mathrm{NT}}(T ; t)\right)=\dot{x}_{\mathrm{C}}(t) e^{r_{1}(T-t)}-r_{1}\left[\left(x_{\mathrm{C}}(t)-x_{p}\right] e^{r_{1}(T-t)}\right. \\
& =\left[\sigma_{\mathrm{C}}(t)-\left(r_{1}+\delta\right) x_{\mathrm{C}}(t)+r_{1} x_{p}\right] e^{r_{1}(T-t)}=\left[\lambda_{\mathrm{C}}(t) / \beta-\left(r_{1}+\delta\right) x_{\mathrm{C}}(t)+r_{1} x_{p}\right] e^{r_{1}(T-t)},
\end{aligned}
$$

where $x_{\mathrm{C}}$ and $\lambda_{\mathrm{C}}$ are given in (38) and (39), while $T$ is obtained from (36). 